\title{
Alterações cardiovasculares em pacientes com SARS-CoV-2 submetidos aos cuidados intensivos: Revisão de escopo
}

Cardiovascular changes in patients with SARS-CoV-2 submitted to intensive care: Scope review Cambios cardiovasculares em pacientes con SARS-CoV-2 presentados a cuidado intensivo: Revisón del alcance

Recebido: 04/02/2021 | Revisado: 13/02/2021 | Aceito: 14/02/2021 | Publicado: 21/02/2021

\author{
Regimar Carla Machado \\ ORCID: https://orcid.org/0000-0001-6126-7663 \\ Universidade Federal de São Paulo, Brasil \\ E-mail: Regimar.carla@unifesp.br \\ João Carlos Marchiori de Claudio \\ ORCID: https://orcid.org/0000-0003-0571-1058 \\ Universidade Federal de São Paulo, Brasil \\ E-mail: joaocarlosmdec@gmail.com \\ Karine Santos do Carmo \\ ORCID: https://orcid.org/0000-0003-0837-9644 \\ Universidade Federal de São Paulo, Brasil \\ E-mail: santosdocarmokarina@gmail.com \\ José Antonio Gonçalves da Silva \\ ORCID: https://orcid.org/0000-0002-7178-9235 \\ Universidade Federal de São Paulo, Brasil \\ E-mail: paraotony@gmail.com \\ Rodrigo Mendes \\ ORCID: https://orcid.org/0000-0001-5712-2111 \\ Universidade Federal de São Paulo, Brasil \\ E-mail: rodrigo_mendes_1996@hotmail.com \\ Sueli Fatima Sampaio \\ ORCID: https://orcid.org/0000-0003-1273-7707 \\ Universidade Federal de São Carlos, Brasil \\ E-mail: sufasampaio@gmail.com
}

\begin{abstract}
Resumo
Objetivo: mapear a literatura científica sobre as alterações cardiovasculares de maior incidência em pacientes com SARS-CoV-2 submetidos às unidades de cuidados intensivos. Método: scoping review seguindo o delineamento do Joanna Briggs Institute. Contemplou as bases de literatura branca e cinzenta, sem restrição temporal e idiomática. Realizadas três etapas de seleção dos estudos: seleção por título e resumo; leitura na íntegra dos estudos selecionados e leitura das referências dos estudos incluídos. Os estudos incluídos foram apresentados em síntese narrativa e tabelas. Resultados: obteve-se um total de 2269 publicações, obtendo oito estudos após as etapas de seleção. Os achados mais relevantes foram: leucocitopenia, aumento de marcadores de necrose cardíaca, alteração do segmentos ST, espessamento do ventrículo esquerdo e redução no fluxo de ejeção do ventrículo esquerdo. Conclusão: as alterações cardíacas com maior incidência encontrada nesses pacientes foram as causadas pela resposta inflamatória no coração e a sobrecarga da bomba cardíaca.
\end{abstract}

Palavras-chave: Revisão de escopo; SARS-CoV-2; Unidade de tratamento intensivo; Alterações cardiovasculares.

\begin{abstract}
Objective: to map the scientific literature on cardiovascular alterations with the highest incidence in patients with SARS-CoV-2 submitted to intensive care units. Method: scoping review following the design of the Joanna Briggs Institute. Contemplated the bases of white and gray literature, without temporal and idiomatic restriction. Three stages of study selection were performed: selection by title and abstract; reading in full the selected studies and reading the references of the included studies. The included studies were presented in narrative synthesis and tables. Results: a total of 2269 publications were obtained, obtaining eight studies after the selection steps. The most relevant findings were: leukocytopenia, increase in cardiac necrosis markers, alteration of the ST segments, thickening of the left ventricle and reduction in left ventricular ejection flow. Conclusion: the cardiac alterations with the highest incidence found in these patients were those caused by the inflammatory response in the heart and the overload of the cardiac pump.
\end{abstract}

Keywords: Scope review; SARS-CoV-2; Intensive care unit; Cardiovascular changes. 


\begin{abstract}
Resumen
Objetivo: mapear la literatura científica sobre las alteraciones cardiovasculares con la mayor incidencia en pacientes con SARS-CoV-2 sometidos a unidades de cuidados intensivos. Método: revisión del alcance siguiendo el diseño del Instituto Joanna Briggs. Contempló las bases de la literatura blanca y gris, sin restricción temporal e idiomática. Se realizaron tres etapas de selección de estudios: selección por título y resumen; leer en su totalidad los estudios seleccionados y leer las referencias de los estudios incluidos. Los estudios incluidos se presentaron en síntesis narrativa y tablas. Resultados: se obtuvieron un total de 2269 publicaciones, obteniéndose ocho estudios después de los pasos de selección. Los hallazgos más relevantes fueron: leucocitopenia, aumento de los marcadores de necrosis cardíaca, alteración de los segmentos ST, engrosamiento del ventrículo izquierdo y reducción del flujo de eyección del ventrículo izquierdo. Conclusión: las alteraciones cardíacas con la mayor incidencia encontrada en estos pacientes fueron las causadas por la respuesta inflamatoria en el corazón y la sobrecarga de la bomba cardíaca.
\end{abstract}

Palabras clave: Revison del alcance; SARS-CoV-2; Unidad de terapia intensiva; Cambios cardiovasculares.

\title{
1. Introdução
}

No final do ano de 2019, uma doença ocasionada por um patógeno não identificado, começou a ser difundida na província de Wuhan, China (Huang, et al, 2020). Pacientes adentravam a emergência apresentando indícios e queixas de um resfriado comum, contudo manifestavam uma severa crise respiratória. Os pacientes com o quadro mais grave necessitam de intubação (Wang, Hu \& Hu, 2020).

Esse patógeno, posteriormente identificado por Coronavírus-19, ou SARS-CoV-2, apresenta similaridade com os vírus causadores da Síndrome Respiratória Aguda Grave (SARS) e a Síndrome Respiratória do Orientes (MERS) (Parry, 2020). Em humanos, liga-se ao receptor do gene ACE-2 (enzima conversora de angiotensina 2), o qual é encontrado em grande expressão em múltiplos órgãos (ex. pulmão, coração, rins) (South et al, 2020). O funcionamento do vírus como "bloqueador" para a ACE-2 causa diversas lesões nos indivíduos, que apresentam uma considerável taxa de internação em Unidade de Terapia Intensiva (UTI) (Hu et al, 2020; Wu, 2020).

Diante da parentalidade entre o vírus da COVID-19 com o causador da SARS, deve-se observar dados clínicos anteriores, os quais constatam, na maior parte dos casos, alterações na função sistólica ( $\mathrm{Li}$ et al, 2020), como também taquicardia nos pacientes acometidos pela SARS (Yu et al, 2020). Mais recentemente, Ruan et al (2020), apresentou dados similares em pacientes com COVID-19, associando a alta taxa de mortalidade desses por complicações cardiovasculares. Em consonância, estudos sugerem alta prevalência de injúria cardíaca ocasionada pelo SARS-CoV-2 em pacientes hospitalizados, sendo mais comumente reportado complicações nas coronárias (Shi et al, 2020), hipertensão (Guo et al, 2020) e a ocorrência de insuficiência cardíaca (Inciardi et al, 2020).

Os efeitos no sistema cardiovascular em pacientes portadores da COVID- 19 são muito complexos, entretanto o rápido avanço das pesquisas contribui para revelar novas evidências sobre a doença. Contudo, ao observar as evidências em pacientes sob cuidados intensivos, constata-se alta representatividade na taxa de mortalidade dessa população (Grasselli, Pesenti \& Cecconi, 2020) Entretanto, ainda são poucos os achados na literatura mundial sobre essa temática, na perspectiva do comportamento da doença, dos agravos à saúde e dos desfechos clínicos. Sendo assim, o objetivo desta scoping review é mapear a literatura científica, as alterações cardiovasculares de maior incidência em pacientes com SARS-CoV-2 internados em unidades de cuidado intensivo.

\section{Metodologia}

Scoping review, seguindo a metodologia do Joanna Briggs Institute (Peters, 2020) e os termos "Preferred Reporting Items for Systematic Reviews and Meta Analyses Extension for Scoping Review" (PRISMA-ScR) (Tricco et al, 2018). Además, protocolada na plataforma Open Science Framework, com o número de registro 10.17605/OSF.IO/TJ6SV. 
Para a elaboração da pergunta da pesquisa, foi utilizado o método mnemônico população - conceito - contexto (PCC) (Peters, 2020). Dessa forma, obteve-se a pergunta focal: quais as alterações cardiovasculares encontradas em pacientes submetidos à Unidade de Terapia Intensiva com diagnóstico positivo para COVID-19?

Como critérios de inclusão, foram considerados os estudos primários disponíveis por completo para acesso dos pesquisadores que apresentaram como amostra pacientes com diagnóstico de COVID-19 submetidos aos cuidados intensivo e estudos que discriminaram os dados dos pacientes nessas condições. Não houve limite temporal ou de idiomas. Os critérios de exclusão dos estudos foram o delineamento metodológico de revisão sistemática sem a população alvo deste estudo, editoriais e cartas ao leitor.

A estratégia de busca foi realizada em três etapas. A primeira etapa contemplou a realização da busca nas bases de dados Medline e Pubmed e dos melhores descritores nas plataformas Descritores em Ciências da Saúde (DeCS), via BVSalud e Medical subject Headings (MeSH), via Pubmed. Com isso, a busca foi realizada por meio da captação das palavras-chave formadas pela estratégia PCC: "COVID-19" e "cardiovascular disease". A partir da combinação dessas com o descritor booleano AND, foram encontrados os demais descritores, os quais são: "Novel coronavirus", "Novel coronavirus 2019”, "2019 nCoV", "Wuhan coronavirus", "Wuhan pneumonia", "wuhan pandemic", "2019-nCoV", "SARS-CoV-2", STEMI, "acute myocardial infarction", "fibrinolysis", "percutaneous coronary intervention", "heart disease".

A segunda etapa de busca foi guiada pela elaboração de uma estratégia com todos os descritores junto aos operadores booleanos AND e OR, como utilizado na base Pubmed: ("Novel coronavirus" OR "Novel coronavirus 2019" OR "2019 nCoV" OR "COVID-19" OR "Wuhan coronavirus" OR "Wuhan pneumonia" OR "Wuhan pandemic" OR "2019-nCoV" OR "SARS-CoV-2") AND (STEMI OR "acute myocardial infarction" OR "fibrinolysis" OR "percutaneous coronary intervention" $O R$ "heart disease" OR "cardiovascular disease"). Essa estratégia foi realizada em diversas bases de dados, como Web of Science, Scopus, EBSCO All Database, EMBASE Cochrane Library, PROQUEST e Mednar. Foi utilizado o fluxograma PRISMA para melhor demonstrar o caminho de seleção dos estudos (Moher, Liberati, Tetzlaff, Altman, 2009).

Todo o processo de seleção, desde a remoção das duplicatas dos artigos foi realizado por meio do software Endnote e por dois revisores independentes (JC, K). Qualquer discordância entre os revisores foi solucionada por meio do parecer de um terceiro revisor (A). Ademais, os artigos que cumpriram os critérios de inclusão tiveram suas referências observadas para encontrar artigos que não foram contemplados pela estratégia inicial.

Os artigos selecionados foram analisados por meio de um instrumento que visava captar o nome do primeiro autor, ano de publicação, revista de publicação, país de publicação, desenho e objetivo do estudo, amostra estudada, alteração cardiológica encontrada e exames clínicos realizados junto aos resultados. Os resultados foram apresentados em formato de tabela e em forma de síntese narrativa, realçando os achados mais pertinentes frente às alterações cardiológicas.

\section{Resultados}

Foram identificados 2269 artigos, além de um artigo encontrado nas referências dos estudos incluídos. A base de dados que apresentou maior quantidade de publicações foi o Mednar, com um total de 1415 publicações. Foram incluídos oito estudos que preencheram os critérios de inclusão desta revisão e foram excluídos 14 estudos. O processo de seleção dos artigos encontra-se ilustrado na Figura 1. 
Figura 1 - Fluxograma de seleção dos estudos incluídos nesta revisão, São Paulo, 2020.
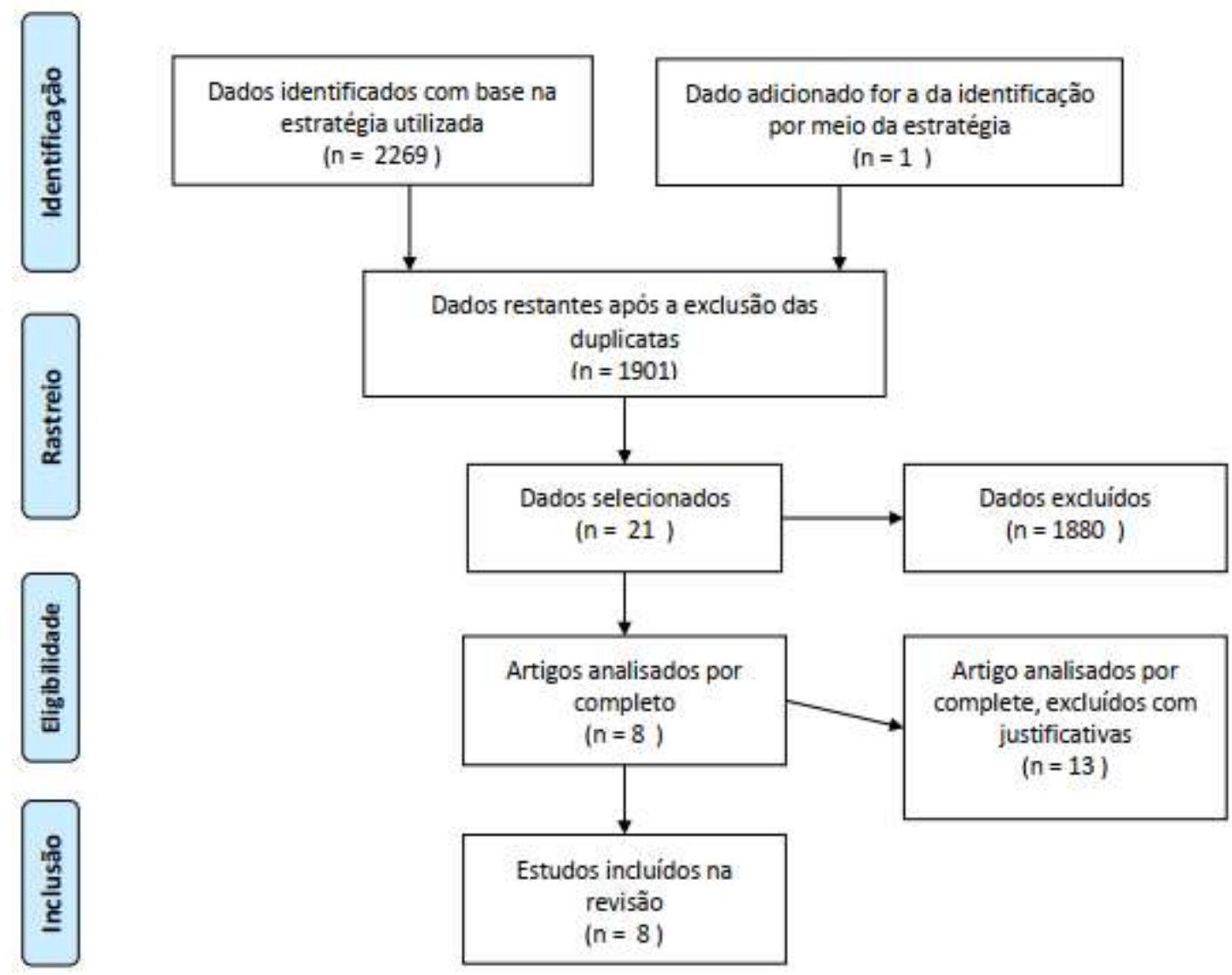

Fonte: Autores

Após finalizar a seleção dos estudos, foi extraído sua origem, a revista onde foi publicado, seu objetivo, sua amostra total e os testes realizados para identificar as alterações cardiovasculares, esses dados são encontrados na Tabela 1.

Tabela 1 - Descrição dos achados nos estudos incluídos nesta revisão, São Paulo, 2020.

\begin{tabular}{|c|c|c|c|c|c|c|}
\hline Autor/Ano & País & Revista & $\begin{array}{l}\text { Delineamento } \\
\text { do estudo }\end{array}$ & Objetivo do estudo & $\begin{array}{l}\text { População do } \\
\text { estudo }\end{array}$ & $\begin{array}{ll}\text { Método } & \text { de } \\
\text { mensuração } & \text { da } \\
\text { alteração } & \end{array}$ \\
\hline $\begin{array}{l}\text { Inciardi et al, } \\
2020\end{array}$ & Itália & $\begin{array}{l}\text { JAMA Cardiology/ } \\
\text { Brief Report }\end{array}$ & $\begin{array}{l}\text { Estudo } \\
\text { caso }\end{array}$ & $\begin{array}{lr}\text { Descrever } & \text { e } \\
\text { apresentar } & \text { as } \\
\text { inflamações agudas } \\
\text { do miocárdio em um } \\
\text { paciente } \\
\text { coronavírus } & \text { com } \\
\text { se recuperou da } & \text { da } \\
\text { síndrome } & \text { e } \\
\text { desenvolveu fadiga e } \\
\text { sinais e sintomas de }\end{array}$ & $\begin{array}{l}\text { Mulher, } \\
\text { branca de } 53 \\
\text { anos que } \\
\text { chegou no } \\
\text { hospital pela } \\
\text { emergência }\end{array}$ & $\begin{array}{l}\text { - Realização de dois } \\
\text { teste virais; } \\
\text { - Confirmação de } \\
\text { alterações cardíacas: } \\
\text { - Ressonância } \\
\text { magnética; } \\
\text {-Eletrocardiograma } \\
\text { (ECG); } \\
\text { - Testes laboratoriais }\end{array}$ \\
\hline
\end{tabular}


Research, Society and Development, v. 10, n. 2, e41010212689, 2021

(CC BY 4.0) | ISSN 2525-3409 | DOI: http://dx.doi.org/10.33448/rsd-v10i2.12689

\begin{tabular}{|c|c|c|c|c|c|c|}
\hline & & & & $\begin{array}{l}\text { insuficiência cardíaca } \\
\text { após semanas após } \\
\text { sintomas de trato } \\
\text { respiratório superior }\end{array}$ & & completos \\
\hline $\begin{array}{l}\text { Yang et al, } \\
2020\end{array}$ & China & The lancet & $\begin{array}{l}\text { Observacional } \\
\text { Retrospectivo }\end{array}$ & $\begin{array}{l}\text { Descrever os cursos e } \\
\text { resultados clínicos de } \\
\text { pacientes críticos com } \\
\text { pneumonia SARS- } \\
\text { Cov-2 }\end{array}$ & $\begin{array}{l}\text { Amostra de } 52 \\
\text { pacientes } \\
\text { internados em } \\
\text { cuidados } \\
\text { críticos, idade } \\
\text { média de } 59,1 \\
(13,3), \quad 67 \% \\
\text { sexo } \\
\text { masculino, } \\
33 \% \text { exposto } \\
\text { ao mercado de } \\
\text { Hunan, } 40 \% \\
\text { com alguma } \\
\text { doença crônica } \\
\text { basal. }\end{array}$ & $\begin{array}{l}\text { - Teste laboratorial } \\
\text { sanguíneo completo; } \\
\text { - Avaliação de sinais } \\
\text { e sintomas no } \\
\text { decorrer } \\
\text { hospitalização }\end{array}$ \\
\hline $\begin{array}{l}\text { Zeng et al. } \\
2020\end{array}$ & China & Infection & $\begin{array}{l}\text { Estudo de } \\
\text { caso }\end{array}$ & $\begin{array}{l}\text { Apresentar as } \\
\text { complicações do } \\
\text { covid19 na miocardite } \\
\text { fulminante }\end{array}$ & $\begin{array}{l}\text { Homem de } 63 \\
\text { anos de idade, } \\
\text { com histórico } \\
\text { de tabagismo, } \\
\text { com histórico } \\
\text { recente de } \\
\text { viagem para a } \\
\text { província de } \\
\text { Hubei }\end{array}$ & $\begin{array}{l}\text { - Realização de dois } \\
\text { teste virais; } \\
\text { - Tomografia } \\
\text { computadorizada; } \\
\text { - Eletrocardiograma } \\
\text { (ECG); } \\
\text { - Testes laboratoriais } \\
\text { completos }\end{array}$ \\
\hline $\begin{array}{l}\text { Juusela, } \\
\text { Nazir, } \\
\text { Gimovsky, } \\
2020\end{array}$ & $\begin{array}{l}\text { Estados } \\
\text { Unidos } \\
\text { da } \\
\text { América }\end{array}$ & $\begin{array}{l}\text { American Journal of } \\
\text { Obsetetrics and } \\
\text { Gynecology MFM }\end{array}$ & $\begin{array}{l}\text { EStudo } \\
\text { Caso }\end{array}$ & $\begin{array}{l}\text { Duas gestantes com } \\
\text { cardiomiopatia e com } \\
\text { coronavírus }\end{array}$ & $\begin{array}{lr}\text { M1: } 45 & \text { anos, } \\
\text { africana, } & 39 \\
\text { semanas } & \text { e } \\
\text { meia } & \text { de } \\
\text { gestação. } & \\
& \\
\text { M2: } 26 & \text { anos, } \\
\text { latina, } & 33 \\
\text { semanas } & \text { de } \\
\text { gestação }\end{array}$ & $\begin{array}{l}\text { M1: } \\
\text { Ecocardiograma, } \\
\text { Proteinúria, } \\
\text { Contagem de } \\
\text { plaquetas, Função } \\
\text { Hepática, Troponina, } \\
\text { BNP, Procalcitonina, } \\
\text { RX Tórax, ECG, } \\
\text { K+, }\end{array}$ \\
\hline
\end{tabular}

M2:Gasometria 


\begin{tabular}{|c|c|c|c|c|c|c|}
\hline & & & & & & $\begin{array}{l}\text { Arterial, RX tórax, } \\
\text { Ácido Lático,PCR, } \\
\text { ferritina, } \\
\text { procalcitonina, } \\
\text { troponina, função } \\
\text { hepática, teste para } \\
\text { Covide, } \\
\text { ecocardiograma, }\end{array}$ \\
\hline $\begin{array}{l}\text { Xiao et al, } \\
2020\end{array}$ & China & $\begin{array}{l}\text { Chinese Journal of } \\
\text { Contemporary } \\
\text { pediatrics }\end{array}$ & $\begin{array}{l}\text { Estudo } \\
\text { caso }\end{array}$ & $\begin{array}{l}\text { Avaliar o tratamento } \\
\text { de pacientes com } \\
\text { infarto agudo do } \\
\text { miocárdio na } \\
\text { pandemia do COVID } \\
19\end{array}$ & $\begin{array}{l}\text { Caso 1: Não } \\
\text { apresenta } \\
\text { diagnóstico de } \\
\text { COVID } 19 \\
\text { Caso } \\
\text { Homem, } 42 \\
\text { anos, residente } \\
\text { de Wuhan. } \\
\text { Histórico de } \\
\text { Hipertensão e } \\
\text { diabetes } \\
\text { Caso 3: Não } \\
\text { apresenta } \\
\text { diagnóstico de } \\
\text { COVID19 }\end{array}$ & $\begin{array}{l}\text { Caso 2: } \\
\text { Eletrocardiograma, } \\
\text { tomografia } \\
\text { computadorizada e } \\
\text { exame de sangue } \\
\text { completo }\end{array}$ \\
\hline $\begin{array}{l}\text { Ma et al, } \\
2020\end{array}$ & China & medRxiv & $\begin{array}{l}\text { Estudo } \\
\text { coorte }\end{array}$ & $\begin{array}{l}\text { Abordar as questões } \\
\text { cardiológicas e seus } \\
\text { fatores severos com } \\
\text { base em } 84 \text { adultos no } \\
\text { hospital de } \\
\text { Chongqing, China }\end{array}$ & $\begin{array}{l}84 \text { adultos } \\
\text { com } \\
\text { diagnóstico de } \\
\text { COVID } 19 \text { (20 } \\
\text { com caso } \\
\text { severo; } 64 \\
\text { sem } \\
\text { complicações } \\
\text { severas) }\end{array}$ & $\begin{array}{l}\text { - Realização de dois } \\
\text { teste virais; } \\
\text { - Tomografia } \\
\text { computadorizada do } \\
\text { peito; } \\
\text {-Eletrocardiograma } \\
\text { (ECG); } \\
\text {-Testes laboratoriais } \\
\text { completos }\end{array}$ \\
\hline $\begin{array}{l}\text { Bhatrajuet al, } \\
2020\end{array}$ & $\begin{array}{l}\text { Estados } \\
\text { unidos } \\
\text { da } \\
\text { América }\end{array}$ & $\begin{array}{l}\text { The new England } \\
\text { Journal of Medicine }\end{array}$ & $\begin{array}{l}\text { Estudo } \\
\text { transversal }\end{array}$ & $\begin{array}{l}\text { Descrever a } \\
\text { características } \\
\text { demográficas, } \\
\text { condições } \\
\text { coexistentes, achados } \\
\text { em imagens e } \\
\text { resultados de }\end{array}$ & $\begin{array}{l}\text { Nove hospitais } \\
\text { com unidades } \\
\text { de cuidados } \\
\text { críticos na } \\
\text { região de } \\
\text { Seattle, } \\
\text { apresentando }\end{array}$ & $\begin{array}{l}\text { - Realização do teste } \\
\text { RT-PCR; } \\
\text { - Radiologia do peito } \\
\text { - } \quad \text { Tomografia } \\
\text { computadorizada, }\end{array}$ \\
\hline
\end{tabular}




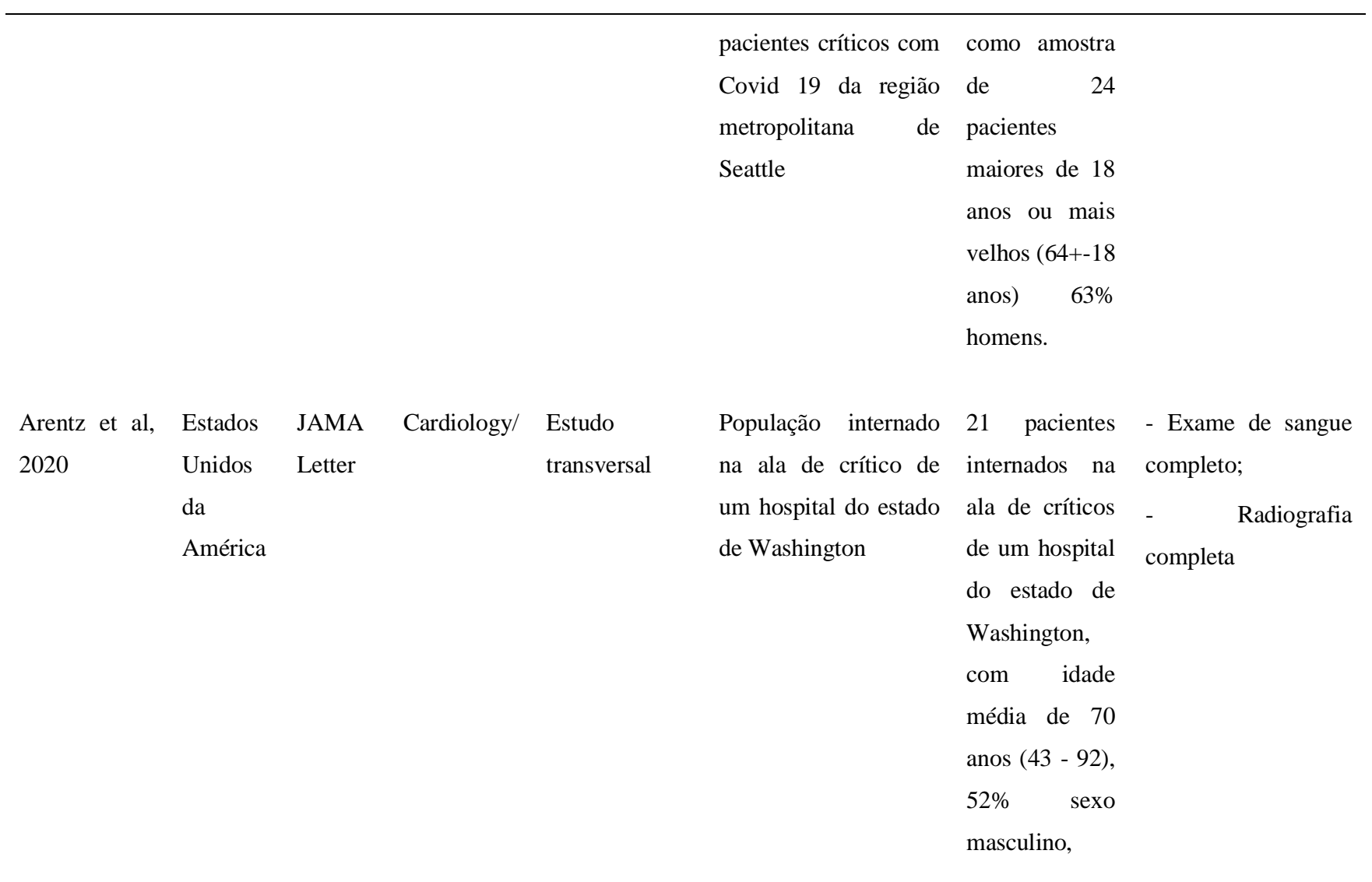

Fonte: Autores

Realizada a extração dos componentes básicos dos artigos selecionados (Tabela 1), foi realizado a extração dos dados clínicos obtidos frete as alterações cardiológicas observadas nos estudos. Mais detalhes sobre os estudos encontram-se na Tabela 2.

Tabela 2 - Alterações clínicas encontradas nos exames realizados pelos estudos, São Paulo, 2020.

\begin{tabular}{|c|c|c|c|c|c|}
\hline Autor/Ano & Exames de sangue & Eletrocardiograma & $\begin{array}{l}\text { Ressonânc } \\
\text { ia } \\
\text { magnética }\end{array}$ & $\begin{array}{l}\text { Exame } \\
\text { Tomografia } \\
\text { computador } \\
\text { izada }\end{array}$ & $\begin{array}{l}\text { Sinais clínicos observados do dano } \\
\text { cardiológico }\end{array}$ \\
\hline $\begin{array}{l}\text { Inciardiet } \\
\text { al, } 2020\end{array}$ & $\begin{array}{l}\text { Elevação dos marcadores de } \\
\text { injúria celular miocárdica: } \\
\text { necrose miosite, Proteína } \\
\text { Creatina, creatinina kinase, } \\
\text { Troponina T e NT - } \\
\text { ProBNP. }\end{array}$ & $\begin{array}{l}\text { Ritmo sinusal com } \\
\text { menor voltagem; } \\
\text { Elevação difusa nos } \\
\text { segmentos ST; } \\
\text { Depressão } \quad \text { do } \\
\text { segmento ST, com } \\
\text { inversão da onda T. }\end{array}$ & $\begin{array}{l}\text { Espessame } \\
\text { nto do } \\
\text { ventrículo } \\
\text { esquerdo, }\end{array}$ & $\begin{array}{l}\text { Não } \\
\text { realizado }\end{array}$ & $\begin{array}{l}\text { Ao ser admitida na emergência } \\
\text { apresentava: dor no peito, dispneia e } \\
\text { febre. No decorrer da internação } \\
\text { apresentou:não consta no artigo }\end{array}$ \\
\hline $\begin{array}{l}\text { Yang et al, } \\
2020\end{array}$ & Constatou leucocitopenia & Não realizado & $\begin{array}{l}\text { Não } \\
\text { realizado }\end{array}$ & $\begin{array}{l}\text { Não } \\
\text { realizado }\end{array}$ & $\begin{array}{l}\text { Sintomas mais comuns nos pacientes: } \\
\text { febre }(98 \%) \text {, Tosse }(77 \%) \text {, Dispneia } \\
(63,5 \%) \text { Mialgia }(11,5 \%) .\end{array}$ \\
\hline $\begin{array}{l}\text { Zeng et al. } \\
2020\end{array}$ & $\begin{array}{l}\text { Elevação nos marcadores: } \\
\text { Troponina T, mioglobina e } \\
\text { NT - ProBNP. }\end{array}$ & \begin{tabular}{l}
\multicolumn{3}{c}{ Taquicardia sinusal e } \\
no segmento ST, \\
junto a um \\
alargamento ro \\
ventrículo esquerdo
\end{tabular} & $\begin{array}{l}\text { Não } \\
\text { realizado }\end{array}$ & $\begin{array}{l}\text { Não } \\
\text { realizado }\end{array}$ & $\begin{array}{l}\text { Não foi observado sinais e sintomas } \\
\text { durante o estudo }\end{array}$ \\
\hline
\end{tabular}


e uma fração de ejeção do ventrículo esquerdo diminuída, e uma hipertensão pulmonar.

Juusela,
Nazir,

Gimovky,

M1: 45

afrodescendente,

anos, RX

2020

semanas de gestação.

Proteinúria 1p, Plaquetas:

274.000 por mcL, AST:

32UI, ALT: 24UI, Gaso

Art: $\mathrm{pH}: 7,27$; pCO2: 31; pO2: 117; HCO3: 16, BE: $11 \mathrm{mmol}$

Teste de Covid positivo, BNP:114, Procalcitonina: $0,13, \mathrm{~K}+3,7$

$2^{\mathrm{a}}$ Tropo: 0,930

com BNP: 323;

M2: 26 anos, latina, 33 semanas de gestação

AST: 47 UI, ALT: 52 UI, Gaso Art: pH: 7,32; pCO2: 18; pO2: 107; HCO3: 14, BE: 14 mmol, acidose metabólica com alcalose respiratória, Painel respiratório geral negativo,

BNP: $>10$

Xiao et al, Caso 2: A contagem de 2020 linfócitos abaixo do normal (na admissão).
Ma et al, Marcadores inflamatórios 2020
PX: Pneumonia Não
Caso 2: Elevação Não das ondas do realizado segmento ST pelos condutores V1-V5 (sugestão de reinfarto anterior)

de

congestiva, ICC

Ecocardiograma:

(FEVE): $\quad 40 \%$ Hipocinesia global, ECG:

Anormalidades inespecíficas da onda T, QTc: 354 de $465 \mathrm{~ms}$,

Ecocardiograma: (FEVE): $\quad 40 \%$ Hipocinesia global,
Não realizado

arritmia cardíaca, Não desvio do eixo realizado esquerdo, Rápida fibrilação atrial, mudança de Ondas $\mathrm{T}$, bloqueio do primeiro degrau atrioventricular,

onda inferiormente anormal, Mudança nas ondas $\mathrm{T}$, batimentos atriais prematura, bloqueio atrioventricular de primeiro grau
Caso 2: Caso 2:Após 3 dias da admissão $\rightarrow$ Achados de Relato de dor no peito fibrose cardíaca e mudança focal por exaustão, sugeridas por pneumonia viral (na admissão).

Índice de Precordial, dispneia, Fadiga, dificuldade inflamação de respirar, palpitações, dor no peito,
Taquicardia,Hipertensão Grave, SATO2: $80 \%$,

Dispnéia, diminuição fetal, Taquicardia,
PCT, IL-6); Marcadores de aumentados (CK aumentados (CK, Alfa- HBHD, IAM); 


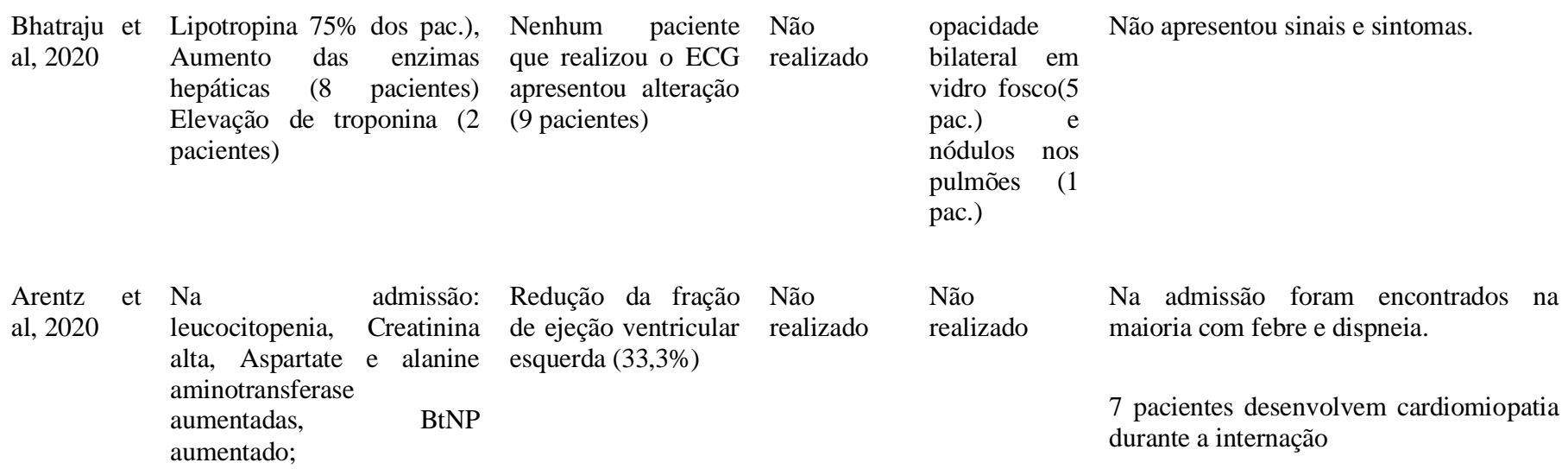

Legendas: BtNP: brain-type natriuretic peptide; Proteina reativa- C (CRP); taxa de sedimentação de eritrócitos (ESR); Procalcitonina (PCT); Interleucina 6 (IL-6); Creatina Kinase (CK); Lactato desidrogenase sérica (LDH); Alfa-Hydroxybutyrate Dehydrogenase (Alfa-HBHD), Albumina modificada por isquemia (IAM); FEVE: Fração de ejeção do ventrículo esquerdo; NT-ProBNP: N-terminal do peptídeo natriurético tipo B. Fonte: Autores.

\section{Discussão}

O objetivo do estudo foi realizar um escopo da literatura que conseguisse demonstrar as alterações cardiovasculares mais incidentes entre os pacientes acometidos pelo SARS-CoV-2, no setor de alta complexidade do hospital ou unidade de cuidado. Observa-se, em primazia, que a maioria dos estudos foram produzidos na China e, logo em seguida, nos Estados Unidos da América (EUA). Esse fato deve-se pela gênese da pandemia no continente asiático, mais especificamente, na província de Wuhan, China (Huang et al, 2020). Por outro lado, o número acentuado de pesquisas desenvolvidas nos EUA, deve-se ao fato de ser o segundo país com maior números de casos atualmente (Who, 2020), apresentando uma vasta gama de material para ser estudado nesse país. Esses dados são complementares a outras revisões que demonstraram o mesmo quantitativo de estudos por localidade (Madjid, Safavi-Naeini, Solomon \& Vardeny, 2020).

Ao avaliar a metodologia utilizada nos estudos, observa-se que a maioria é constituída por estudos de caso, uma hipótese para esse fenômeno é que essa metodologia avalia o paciente em um momento único, descrevendo todos os achados possíveis, de forma mais rápida e completa para que a publicação seja submetida (Creswell, 2010). Contudo, para obter mais detalhes sobre o trajeto da evolução dos pacientes, seria de interesse a utilização de estudos com metodologia observacional ou coorte, pois apresentam mais informações a respeito do tempo de hospitalização e contém maior número de pacientes avaliados.

Outro ponto relevante dos estudos é a utilização de exames complementares para o diagnóstico, pois é possível observar uma tríade de exames comum: eletrocardiograma, exames de sangue completo e tomografia computadorizada. Esses exames são os mais prevalentes por possibilitarem a análise completa do paciente.

Como exemplo, o exame de sangue possibilita a análise dos fatores sanguíneos e seus componentes, conseguindo diagnosticar com mais facilidade alterações de marcadores sanguíneos. Frente a esses achados nos estudos incluídos, foi possível observar um aumento dos marcadores inflamatórios (CRP, ESR, PCT e IL-6), com baixíssima incidência de corpos virais. Sugere-se, como demonstrado na literatura basilar, a possibilidade de replicação viral no tecido do coração (miocardite), como também uma resposta imune de citotoxinas para desempenhar o papel de combate. Esse resultado pode ser complementado pela presença do aumento das enzimas cardíacas (CK, LDH, Alfa-HBHD e IAM) e marcadores de necrose (miosite) (Esfandiari, 2008). A alteração dos marcadores pode desencadear uma disfunção ventricular, respondendo assim o sintoma de dor no peito, relatado em parte dos estudos incluídos (Yang et al, 2020). 
Outro exame utilizado foi o eletrocardiograma, o qual possibilita a análise do ritmo sinusal e da condução elétrica do coração. Sobre os achados dos estudos incluídos, apresenta-se com maior incidência as arritmias cardíacas, desvio do eixo esquerdo, elevação difusa no segmento ST e a depressão do segmento ST com inversão da onda T. Essas anomalias, como também outras encontradas, podem complementar os achados clínicos expostos pela miocardite, causada pelo coronavírus. Os profissionais de saúde que observarem essas alterações cardiológicas em pacientes com suspeita de infecção do COVID-19, devem sempre empregar cuidados de segurança por meio dos Equipamentos de Proteção Individual (EPIs), a fim de garantir a não contaminação paciente - profissional. O uso de máscaras N95 ou cirúrgicas, junto ao uso de luvas e aventais descartáveis, deve ser adotado, como também a higiene das mãos e o não compartilhamento de objetos de uso pessoal nos pacientes (Who, 2020, China, 2020).

O ecocardiograma foi observado em quatro estudos desta revisão (Zeng et al, 2020; Juusela, Nazir \& Gimovsky, 2020; Bhatraju et al, 2020; Arentz et al, 2020), dessa forma, observou-se, como alterações cardiológicas mais comuns, a redução na fração de ejeção do ventrículo esquerdo, falha na ejeção e miocardite fulminante. Esses dados estão em consonância com os sintomas já mencionados causados pelo SARS-Cov-2, pois o vírus gera inflamação no miocárdio e a destruição celular muscular faz com que a bomba cardíaca reduza sua evasão sanguínea e a falha na ejeção. Da mesma forma, a miocardite fulminante é explicada pelo mesmo patógeno, SARS-CoV-2, o qual realiza a inflamação no músculo cardíaco e gera complicações como o supradesnivelamento do segmento ST-T e a dor precordial (Dec et al, 1985; Dec et al, 1992; SBC, 2008; Babapoor et al, 2020). Um estudo, dentre os incluídos, não apresentou modificação ao realizar esse exame (Bhatraju et al, 2020). Contudo, deve-se observar que esse estudo apresentou somente nove pacientes do total de 24 de sua amostra, que foram testados apenas uma única vez, pois a metodologia empregada foi descrita como transversal.

Ademais, o exame de ressonância magnética, foi descrito em apenas em um estudo (Inciardi et al, 2020) e apresentou como resultado o espessamento do ventrículo esquerdo. O que demonstra uma compensação muscular exigida pela falha descrita anteriormente, ocasionada pela insuficiência cardíaca gerada pela redução da ejeção (Lopes \& Liberatori, 1998; Babapoor et al, 2020).

Finalizando o quadro de exames utilizados, a tomografia computadorizada foi pouco recorrente (Zeng et al, 2020; Bhatraju et al, 2020; Arentz 2020), mesmo que ela possibilite a avaliação da alteração da morfologia do coração. Os achados apontaram opacidade bilateral em vidro fosco, nódulos pulmonares, inflamação pulmonar e fibrose cardíaca. Atualmente, considera-se que esse padrão possa ser encontrado nas fases agudas ou crônicas de doenças que comprometem o interstício e/ou os alvéolos das mais variadas naturezas. A extensão, distribuição, forma de apresentação e associação com outros achados tomográficos são condições que, quando associadas à clínica, podem caracterizar doenças específicas em determinadas fases do seu curso (Santos et al, 2003).

Ainda, a sintomatologia apresentada nos estudos, foi em maioria identificada como dor no peito, fadiga, dificuldade de respirar, dispneia e febre. Os sintomas anteriores foram considerados comuns para a classificação de pacientes, antes do diagnóstico por exames laboratoriais de coronavírus, como consta no relatório da comissão nacional de saúde chinesa (China, 2020). Porém, quando o paciente é admitido em um leito de alta complexidade, observa-se alterações mais graves, como linfocitopenia e o desenvolvimento de doença renal crônica. Esses sintomas estão sendo apresentados como causas complementares para a internação (Guan et al, 2020). A linfocitopenia, de acordo com a literatura, relaciona-se à família do vírus SARS-CoV que causa danos no componente citoplasmático dos linfócitos, gerando a destruição desses (Chu et al, 2020). Esses dados foram relatados de forma semelhante em pacientes que desenvolveram infecções por MERS (Liu et al, 2017).

Frente a grande sintomatologia apresentada pela COVID-19, pesquisas do mundo têm mostrado o aumento de diagnóstico rápido e preciso que possa direcionar as condutas nos cuidados ao paciente. Sabe-se que o alvo desse vírus é o sistema respiratório, sendo assim, há grande importância de exames de imagem radiológica e tomográfica (Acr, 2020). 
Contudo, grande partes dos achados deste estudo apresentou somatória de exames complementares que auxiliam não no diagnóstico, mas sim no tratamento e na busca da melhor conduta para as possíveis complicações que o paciente apresentava, sendo assim, deve-se repensar a adoção desses exames como recursos possíveis para complementar e melhorar o prognóstico do paciente.

Ressalta-se a limitação deste estudo, como o período de rastreio dos estudos, pois a temática vem sendo estudada e renovada diariamente, o que acarreta a exclusão de artigos lançados após o encerramento da busca.

Em contraponto, houve inclusão de uma ampla gama de descritores e bases de dados para se arrecadar o máximo de estudos frente a temática desejada. Junto a isso, a utilização de uma metodologia robusta e criteriosa frente aos estudos que foram captados e selecionados colabora com a confiabilidade ao estudo.

Este estudo fornece uma gama de auxílio para os profissionais da saúde frente ao atendimento aos pacientes positivos para SARS-CoV-2 que forem encaminhados para o setor de cuidado intensivo. Para os profissionais em geral, deve-se entender este estudo como um facilitador de identificação de complicações que possam surgir no tratamento desse paciente. Já para os profissionais de enfermagem, as evidências levantadas ajudam a organização e preparo dos cuidados futuros e, ao mesmo tempo, ajuda na identificação dos agravos que pacientes podem apresentar no decorrer de sua doença.

\section{Conclusão}

Conseguiu-se observar como alterações mais comuns nos exames, o aumento de marcadores inflamatórios - como exemplo IL-6 - e leucocitopenia (nos exames de sangue), elevação ou depressão na onda ST (nos exames de eletrocardiograma), o diminuição da fração de ejeção do ventrículo esquerdo (nos exames de ecocardiograma) e fibrose cardíaca (no exame de tomografia computadorizada).

Para além do que foi descrito, deve-se ter como recomendações futuras pesquisas a continuidade da busca exaustiva nas mais diversas bases cientificas, para conseguir uma captação de artigos mais ampla e que traga maior número de evidencias possíveis. Como também, a utilização de uma metodologia robusta para a seleção dos artigos, essa com a finalidade de apresentar normas e regras que são claras e simples para sua compreensão e reprodução. E, por fim, a atualização constante desta pesquisa, devido a ser um tema que é amplamente estudado atualmente e que deve ser atualizado, visto que é de utilidade publica evidencias como essas para sua repercussão na prática assistencial.

\section{Referências}

Acr Recommendations for the use of Chest Radiography and Computed Tomography (CT) for Suspected COVID-19 Infection. \& Harrison X. Bai e cols, Performance of radiologists in differentiating COVID-19 from viral pneumonia on chest CT Disponível em: https://www.acr.org/Advocacy-andEconomics/ACR-Position-tatements/Recommendations-for-Chest-Radiography-and-CT-for-Suspected-COVID19

Infection/https://pubs.rsna.org/doi/10.1148/radiol.2020200823."

Arentz, M., Yim, E., Klaff, L., Lokhandwala, S., Riedo, F. X., Chong, M., \& Lee, M. (2020). Characteristics and Outcomes of 21 Critically Ill Patients With COVID-19 in Washington State. JAMA, 323(16), 1612-1614. https://doi.org/10.1001/jama.2020.4326

Babapoor-Farrokhran, S., Gill, D., Walker, J., Rasekhi, R. T., Bozorgnia, B., \& Amanullah, A. (2020). Myocardial injury and COVID-19: Possible mechanisms. Life sciences, 253, 117723. https://doi.org/10.1016/j.lfs.2020.117723

Bhatraju, P. K., Ghassemieh, B. J., Nichols, M., Kim, R., Jerome, K. R., Nalla, A. K., Greninger, A. L., Pipavath, S., Wurfel, M. M., Evans, L., Kritek, P. A., West, T. E., Luks, A., Gerbino, A., Dale, C. R., Goldman, J. D., O'Mahony, S., \& Mikacenic, C. (2020). Covid-19 in Critically Ill Patients in the Seattle Region - Case Series. The New England journal of medicine, 382(21), 2012-2022. https://doi.org/10.1056/NEJMoa2004500

China, Chinese Center for Disease Control and Prevention.JAMA. 10.1001/jama.2020.2648

Chinas NHCo. New coronavirus pneumonia prevention and control program (5a ed.). http://www.nhc.gov.cn/yzygj/s7653p/202002/ 3b09b894ac9b4204a79db5b8912d4440"

Chu, H., Zhou, J., Wong, B. H., Li, C., Chan, J. F., Cheng, Z. S., Yang, D., Wang, D., Lee, A. C., Li, C., Yeung, M. L., Cai, J. P., Chan, I. H., Ho, W. K., To, K. K., Zheng, B. J., Yao, Y., Qin, C., \& Yuen, K. Y. (2016). Middle East Respiratory Syndrome Coronavirus Efficiently Infects Human Primary T 
Lymphocytes and Activates the Extrinsic and Intrinsic Apoptosis Pathways. The Journal of infectious diseases, $213(6)$, 904-914. https://doi.org/10.1093/infdis/jiv380

Creswell J. W. Projeto de pesquisa: métodos qualitativo, quantitativo e misto. $3^{\text {a }}$ ed. Porto Alegre (RS): Artmed; 2010

Dec, G. W., Jr, Palacios, I. F., Fallon, J. T., Aretz, H. T., Mills, J., Lee, D. C., \& Johnson, R. A. (1985). Active myocarditis in the spectrum of acute dilated cardiomyopathies. Clinical features, histologic correlates, and clinical outcome. The New England journal of medicine, 312(14), 885-890. https://doi.org/10.1056/NEJM198504043121404

Dec, G. W., Jr, Waldman, H., Southern, J., Fallon, J. T., Hutter, A. M., Jr, \& Palacios, I. (1992). Viral myocarditis mimicking acute myocardial infarction. Journal of the American College of Cardiology, 20(1), 85-89. https://doi.org/10.1016/0735-1097(92)90141-9

Deng, Q., Hu, B., Zhang, Y., Wang, H., Zhou, X., Hu, W., Cheng, Y., Yan, J., Ping, H., \& Zhou, Q. (2020). Suspected myocardial injury in patients with COVID-19: Evidence from front-line clinical observation in Wuhan, China. International journal of cardiology, 311, 116-121. https://doi.org/10.1016/j.ijcard.2020.03.087

Dhawan et al COVID-19 and cardiovascular disease: What we know, what we think we know, and what we need to know, Journal of Molecular and Cellular Cardiology, 2019, DOI: 10.1016/j.yjmcc.2020.04.026

Esfandiarei, M., \& McManus, B. M. (2008). Molecular biology and pathogenesis of viral myocarditis. Annual review of pathology, 3, 127-155. https://doi.org/10.1146/annurev.pathmechdis.3.121806.151534

G Guo, T., Fan, Y., Chen, M., Wu, X., Zhang, L., He, T., Wang, H., Wan, J., Wang, X., \& Lu, Z. (2020). Cardiovascular Implications of Fatal Outcomes of Patients With Coronavirus Disease 2019 (COVID-19). JAMA cardiology, 5(7), 811-818. https://doi.org/10.1001/jamacardio.2020.1017

Grasselli, G., Pesenti, A., \& Cecconi, M. (2020). Critical Care Utilization for the COVID-19 Outbreak in Lombardy, Italy: Early Experience and Forecast During an Emergency Response. JAMA, 323(16), 1545-1546. https://doi.org/10.1001/jama.2020.4031

Guan W, Ni Z, Hu Y, et al. Clinical characteristics of coronavirus disease 2019 in China. N Engl J Med. 10.1056/NEJMoa2002032.

Hu, Y., Sun, J., Dai, Z., Deng, H., Li, X., Huang, Q., Wu, Y., Sun, L., \& Xu, Y. (2020). Prevalence and severity of corona virus disease 2019 (COVID-19): A systematic review and meta-analysis. Journal of clinical virology: the official publication of the Pan American Society for Clinical Virology, $127,104371$. https://doi.org/10.1016/j.jcv.2020.104371.

Huang, C., Wang, Y., Li, X., Ren, L., Zhao, J., Hu, Y., Zhang, L., Fan, G., Xu, J., Gu, X., Cheng, Z., Yu, T., Xia, J., Wei, Y., Wu, W., Xie, X., Yin, W., Li, H., Liu, M., Xiao, Y., Cao, B. (2020). Clinical features of patients infected with 2019 novel coronavirus in Wuhan, China. Lancet (London, England), 395(10223), 497-506. https://doi.org/10.1016/S0140-6736(20)30183-5

Inciardi, R. M., Lupi, L., Zaccone, G., Italia, L., Raffo, M., Tomasoni, D., Cani, D. S., Cerini, M., Farina, D., Gavazzi, E., Maroldi, R., Adamo, M., Ammirati, E., Sinagra, G., Lombardi, C. M., \& Metra, M. (2020). Cardiac Involvement in a Patient With Coronavirus Disease 2019 (COVID-19). JAMA cardiology, 5(7), 819-824. https://doi.org/10.1001/jamacardio.2020.1096

Juusela, A., Nazir, M., \& Gimovsky, M. (2020). Two cases of coronavirus 2019-related cardiomyopathy in pregnancy. American journal of obstetrics \& gynecology MFM, 2(2), 100113. https://doi.org/10.1016/j.ajogmf.2020.100113

Li, J. W., Han, T. W., Woodward, M., Anderson, C. S., Zhou, H., Chen, Y. D., \& Neal, B. (2020). The impact of 2019 novel coronavirus on heart injury: A Systematic review and Meta-analysis. Progress in cardiovascular diseases, 63(4), 518-524. https://doi.org/10.1016/j.pcad.2020.04.008

Li, S. S., Cheng, C. W., Fu, C. L., Chan, Y. H., Lee, M. P., Chan, J. W., \& Yiu, S. F. (2003). Left ventricular performance in patients with severe acute respiratory syndrome: a 30-day echocardiographic follow-up study. Circulation, 108(15), 1798-1803. https://doi.org/10.1161/01.CIR.0000094737.21775.32.

Li, T.-W. Han, M. Woodward, et al., The impact of 2019 novel coronavirus on heart injury: A systematic review and Meta-analysis, Progress in Cardiovascular Diseases (2020), https://doi.org/10.1016/j.pcad.2020.04.008

Liu, W. J., Zhao, M., Liu, K., Xu, K., Wong, G., Tan, W., \& Gao, G. F. (2017). T-cell immunity of SARS-CoV: Implications for vaccine development against MERS-CoV. Antiviral research, 137, 82-92. https://doi.org/10.1016/j.antiviral.2016.11.006

Long, W.J. Brady, A. Koyfman, et al., Cardiovascular complications in COVID-19, American Journal of Emergency Medicine, https://doi.org/10.1016/j.ajem.2020.04.04845.

Lopes A.C., Liberatori Filho A. W. Tratamento da insuficiência cardíaca -- aspectos atuais. Rev. Assoc. Med. Bras. [Internet]. 1998 June [cited 2020 May 16] ; $44(2$ ) : 75-76. http://www.scielo.br/scielo.php?script=sci_arttext\&pid=S0104-42301998000200001\&lng=en.https://doi.org/10.1590/S010442301998000200001 .

Ma et al, COVID-19 Myocarditis and Severity Factors: An Adult Cohort Study medRxiv $2020 . \quad 03 . \quad 19.20034124$; doi: https://doi.org/10.1101/2020.03.19.20034124"

Ma, L., Song, K., \& Huang, Y. (2020). Coronavirus Disease-2019 (COVID-19) and Cardiovascular Complications. Journal of cardiothoracic and vascular anesthesia, S1053-0770(20)30400-6. Advance online publication. https://doi.org/10.1053/j.jvca.2020.04.041

Madjid, M., Safavi-Naeini, P., Solomon, S. D., \& Vardeny, O. (2020). Potential Effects of Coronaviruses on the Cardiovascular System: A Review. JAMA cardiology, 5(7), 831-840. https://doi.org/10.1001/jamacardio.2020.1286

Mahmud, E., Dauerman, H. L., Welt, F. G., Messenger, J. C., Rao, S. V., Grines, C., Mattu, A., Kirtane, A. J., Jauhar, R., Meraj, P., Rokos, I. C., Rumsfeld, J. S., \& Henry, T. D. Management of Acute Myocardial Infarction During the COVID-19 Pandemic, Journal of the American College of Cardiology (2020), https://doi.org/10.1016/j.jacc.2020.04.039. 
Mehra, M. R., Desai, S. S., Kuy, S., Henry, T. D., \& Patel, A. N. (2020). Cardiovascular Disease, Drug Therapy, and Mortality in Covid-19. The New England journal of medicine, 382(25), e102. https://doi.org/10.1056/NEJMoa2007621

Moher, D., Liberati, A., Tetzlaff, J., Altman, D. G., \& PRISMA Group (2009). Preferred reporting items for systematic reviews and meta-analyses: the PRISMA statement. PLoS medicine, 6(7), e1000097. https://doi.org/10.1371/journal.pmed.1000097

Parry J. (2020). Wuhan: Britons to be evacuated as scientists estimate 44000 cases of 2019-nCOV in the city. BMJ (Clinical research ed.), 368 , m351. https://doi.org/10.1136/bmj.m351.

Peiris, J. S., Chu, C. M., Cheng, V. C., Chan, K. S., Hung, I. F., Poon, L. L., Law, K. I., Tang, B. S., Hon, T. Y., Chan, C. S., Chan, K. H., Ng, J. S., Zheng, B. J., Ng, W. L., Lai, R. W., Guan, Y., Yuen, K. Y., \& HKU/UCH SARS Study Group (2003). Clinical progression and viral load in a community outbreak of coronavirus-associated SARS pneumonia: a prospective study. Lancet (London, England), 361(9371), 1767-1772. https://doi.org/10.1016/s01406736(03)13412-5

Peters, M. D., Godfrey, C. M., Khalil, H., McInerney, P., Parker, D., \& Soares, C. B. (2015). Guidance for conducting systematic scoping reviews. International journal of evidence-based healthcare, 13(3), 141-146. https://doi.org/10.1097/XEB.0000000000000050.

Ruan, Q., Yang, K., Wang, W., Jiang, L., \& Song, J. (2020). Clinical predictors of mortality due to COVID-19 based on an analysis of data of 150 patients from Wuhan, China. Intensive care medicine, 46(5), 846-848. https://doi.org/10.1007/s00134-020-05991-x.

Santos et al - Radiol. Brasil.36(6) (2003).

Shi, S., Qin, M., Shen, B., Cai, Y., Liu, T., Yang, F., Gong, W., Liu, X., Liang, J., Zhao, Q., Huang, H., Yang, B., \& Huang, C. (2020). Association of Cardiac Injury With Mortality in Hospitalized Patients With COVID-19 in Wuhan, China. JAMA cardiology, 5(7), 802-810. https://doi.org/10.1001/jamacardio.2020.0950.

Singh, S., \& Desai, R. (2020). COVID-19 and new-onset arrhythmia. Journal of arrhythmia, 36(3), 547-548. https://doi.org/10.1002/joa3.12354

Sociedade Brasileira de Cardiologia. Como tratar. Barueri, SP: Manole; 2008. 2, 424-39.

South, A. M., Diz, D. I., \& Chappell, M. C. (2020). COVID-19, ACE2, and the cardiovascular consequences. American journal of physiology. Heart and circulatory physiology, 318(5), H1084-H1090. https://doi.org/10.1152/ajpheart.00217.2020

Stefanini, G. G., Montorfano, M., Trabattoni, D., Andreini, D., Ferrante, G., Ancona, M., Metra, M., Curello, S., Maffeo, D., Pero, G., Cacucci, M., Assanelli, E., Bellini, B., Russo, F., Ielasi, A., Tespili, M., Danzi, G. B., Vandoni, P., Bollati, M., Barbieri, L., Chieffo, A. (2020). ST-Elevation Myocardial Infarction in Patients With COVID-19: Clinical and Angiographic Outcomes. Circulation, 141(25), 2113-2116. https://doi.org/10.1161/CIRCULATIONAHA.120.047525

Tersalvi, G., Vicenzi, M., Calabretta, D., Biasco, L., Pedrazzini, G., \& Winterton, D. (2020). Elevated Troponin in Patients With Coronavirus Disease 2019: Possible Mechanisms. Journal of cardiac failure, 26(6), 470-475. https://doi.org/10.1016/j.cardfail.2020.04.009

Tricco, A. C., Lillie, E., Zarin, W., O'Brien, K. K., Colquhoun, H., Levac, D., Moher, D., Peters, M., Horsley, T., Weeks, L., Hempel, S., Akl, E. A., Chang, C., McGowan, J., Stewart, L., Hartling, L., Aldcroft, A., Wilson, M. G., Garritty, C., Lewin, S., ... Straus, S. E. (2018). PRISMA Extension for Scoping Reviews (PRISMA-ScR): Checklist and Explanation. Annals of internal medicine, 169(7), 467-473. https://doi.org/10.7326/M18-0850

Wang, D., Hu, B., Hu, C., Zhu, F., Liu, X., Zhang, J., Wang, B., Xiang, H., Cheng, Z., Xiong, Y., Zhao, Y., Li, Y., Wang, X., \& Peng, Z. (2020). Clinical Characteristics of 138 Hospitalized Patients With 2019 Novel Coronavirus-Infected Pneumonia in Wuhan, China. JAMA, 323(11), 1061-1069. https://doi.org/10.1001/jama.2020.1585

"Who, World Health Organization. Coronavirus disease 2019 (COVID-19): situation report $114 . \quad$ https://www.who.int/docs/defaultsource/coronaviruse/situation-reports/20200513-covid-19-sitrep-114.pdf?sfvrsn=17ebbbe_4"

Who, World Health Organization. Coronavirus disease 2019 (COVID-19): situation report $114 . \quad$ https://www.who.int/docs/defaultsource/coronaviruse/situation-reports/20200513-covid-19-sitrep-114.pdf?sfvrsn=17ebbbe_4

Wu, Z., \& McGoogan, J. M. (2020). Characteristics of and Important Lessons From the Coronavirus Disease 2019 (COVID-19) Outbreak in China: Summary of a Report of 72314 Cases From the Chinese Center for Disease Control and Prevention. JAMA, 323(13), 1239-1242. https://doi.org/10.1001/jama.2020.2648.

Xiao, Z., Xu, C., Wang, D., \& Zeng, H. (2020). The experience of treating patients with acute myocardial infarction under the COVID-19 epidemic. Catheterization and cardiovascular interventions: official journal of the Society for Cardiac Angiography \& Interventions, 10.1002/ccd.28951. Advance online publication. https://doi.org/10.1002/ccd.28951

Yang et al. Clinical course and outcomes of critically ill patients with SARS-CoV-2 pneumonia in Wuhan, China: a single-centered, retrospective, observational study. Lancet Respir Med. Published online February 24, 2020. 10.1016/S2213-2600(20)30079-5

Yu, C. M., Wong, R. S., Wu, E. B., Kong, S. L., Wong, J., Yip, G. W., Soo, Y. O., Chiu, M. L., Chan, Y. S., Hui, D., Lee, N., Wu, A., Leung, C. B., \& Sung, J. J. (2006). Cardiovascular complications of severe acute respiratory syndrome. Postgraduate medical journal, 82(964), 140-144. https://doi.org/10.1136/pgmj.2005.037515.

Zeng, J. H., Liu, Y. X., Yuan, J., Wang, F. X., Wu, W. B., Li, J. X., Wang, L. F., Gao, H., Wang, Y., Dong, C. F., Li, Y. J., Xie, X. J., Feng, C., \& Liu, L. (2020). First case of COVID-19 complicated with fulminant myocarditis: a case report and insights. Infection, 48(5), 773-777. https://doi.org/10.1007/s15010020-01424-5. 